\title{
Regularity and Global Structure of Solutions to Hamilton-Jacobi equations II. Convex initial data
}

\author{
YINCHUAN ZHAO \\ LMAM and School of Mathematical Sciences, Peking University \\ Beijing 100871, P. R. China \\ zyc747250@hotmail.com \\ TAO TANG \\ Department of Mathematics, Hong Kong Baptist University \\ Kowloon Tong Hong Kong \\ and \\ Institute of Computational Mathematics \\ Chinese Academy of Sciences, Beijing, China \\ ttang@math.hkbu.edu.hk \\ JINGHUA WANG \\ Institute of Systems Sciences, Academy of Mathematics and System Sciences \\ The Chinese Academy of Sciences, Beijing 100080, P. R. China \\ jwang@amss.ac.cn
}

\begin{abstract}
The paper is concerned with the Hamilton-Jacobi (HJ) equations of multidimensional space variables with convex initial data and general Hamiltonians. Using Hopf's formula (II), we will study the differentiability of the HJ solutions. For any given point,we give a sufficient and necessary condition such that the solutions are $C^{k}$ smooth in some neighborhood of this point. We also study the characteristics of the equations which play important roles in our analysis. It is shown that there are only two kinds of characteristics, one never touches the singularity point, but the other one touches the singularity point in a finite time. Based on these results, we study the global structure of the set of singularity points for the solutions. It is shown that there exists a one-to-one correspondence between the path connected components of the set of singularity points and path connected component of the set $\left\{(D g(y), H(D g(y))) \mid y \in \mathbb{R}^{n}\right\}$ $\backslash\left\{(D g(y), \operatorname{conv} H(D g(y))) \mid y \in \mathbb{R}^{n}\right\}$, where convH is the convex hull of $H$. A path connected component of the set of singularity points never terminates as $t$ increases. Moreover, our results depend only on $H$ and its domain of definition.
\end{abstract}

Keywords: Hamilton-Jacobi equations; Hopf's formula (II); global structure; singularity point.

Mathematics Subject Classification 2000: 35L60, 49L25, 35L67, 35D10. 


\section{Introduction}

Consider the Cauchy problem for the following Hamilton-Jacobi equation:

$$
\left\{\begin{aligned}
u_{t}+H(D u) & =0 & & \text { in } \mathbb{R}^{n} \times(0, \infty), \\
u & =g & & \text { on } \mathbb{R}^{n} \times\{t=0\},
\end{aligned}\right.
$$

where the Hamiltonian $H: \mathbb{R}^{n} \rightarrow \mathbb{R}$ is $C^{k}(k \geq 2)$ with its domain of definition $\left\{D g(y) \mid y \in \mathbb{R}^{n}\right\}$ denoted as $D g\left(\mathbb{R}^{n}\right) ; g: \mathbb{R}^{n} \rightarrow \mathbb{R}$ is $C^{k}(k \geq 2)$ and satisfies

$$
\begin{aligned}
& D^{2} g(y)>0, \forall y \in \mathbb{R}^{n}, \\
& \sup _{y \in \mathbb{R}^{n}}|D g(y)|=M<\infty,
\end{aligned}
$$

$D g\left(\mathbb{R}^{n}\right)$ is convex.

Moreover, let

$$
\begin{aligned}
& G(x, t, y)=g(y)+(x-y) \cdot D g(y)-t H(D g(y)), \\
& \bar{G}(x, t, p)=x \cdot p-g^{*}(p)-t H(p),
\end{aligned}
$$

where $g^{*}$ is the Legendre transform of $g$.

It is known $[2,5,6]$ that the solution to (1.1) is given by Hopf's Formula (II):

$$
u(x, t)=\max _{p \in \Omega} \bar{G}(x, t, p),
$$

where $\Omega=\left\{p \in \mathbb{R}^{n} \mid g^{*}(p)<\infty\right\}$.

Bardi and Evans [2] demonstrated that Hopf's formula (II) gave the unique solution of (1.1) in the "viscosity" sense introduced by Crandall and Lions [4], under the hypotheses

$$
\left\{\begin{array}{l}
H: \mathbb{R}^{n} \rightarrow \mathbb{R} \text { is continuous, } \\
g: \mathbb{R}^{n} \rightarrow \mathbb{R} \text { is uniformly Lipschitz and convex. }
\end{array}\right.
$$

Note that $D g$ is one-to-one, an equivalent formula will be also shown:

$$
u(x, t)=\max _{y \in \mathbb{R}^{n}} G(x, t, y)
$$

In general, the solution $u(x, t)$ defined by the Hopf's formula (II) is not in class $C^{1}$, and its gradient may present a discontinuity at some points. We call a point a singularity point if it is non-differentiable point of the solution $u(x, t)$ or a cluster point of non-differentiable points of the solution $u(x, t)$.

Many authors have established existence and uniqueness theorems of generalized solution (Lipschitz, viscosity, weak solution), yet only few works have been concerned with the differentiability of the solution. Hoang [6] studied the differentiability of the generalized solution for HJ equation. In [?], we have studied the regulaity and global structure of solutions to HJ equations with convex Hamiltonian. It is natural to ask how it is for HJ equations with convex initial data and general Hamiltonians.

Let $U$ be the set that consists of all points $(x, t)$ such that $G(x, t, \bullet)$ has a unique non-degenerate maximizing point. Then $U$ is open on which the solution is 
$C^{k}$ smooth. We study the properties of characteristics, which play important role in our analysis. They are also interesting in their own sake and have other applications. Given $y_{0} \in \mathbb{R}^{n}$, let

$$
C=\left\{(x, t) \mid x=y_{0}+t D H\left(D g\left(y_{0}\right)\right), t>0\right\} .
$$

A characteristic segment $\bar{C}=C \bigcap\{0<t \leq T<\infty\}$ (respectively, a characteristic $C)$ is called valid if $y_{0}$ is a maximizing point for $G(x, t, \bullet)$ for each $(x, t) \in \bar{C}$ (respectively, $C)$. In case $\sup T<\infty$ we prove there exists a point $\left(x_{s}\left(y_{0}\right), t_{s}\left(y_{0}\right)\right)$, where

$$
\left.t_{s}\left(y_{0}\right)=\sup T, \quad x_{s}\left(y_{0}\right)=y_{0}+t_{s}\left(y_{0}\right) D H\left(D g\left(y_{0}\right)\right)\right),
$$

such that $y_{0}$ is a unique degenerate maximizing point or one of the maximizing points for $G\left(x_{s}(y), t_{s}(y), \bullet\right)$, while $y_{0}$ will be no longer a maximizing point for $G(x, t, \bullet)$ for $(x, t) \in C$ and $t>t_{s}\left(y_{0}\right)$ and $y_{0}$ is a unique non-degenerate maximizing point for $G(x, t, \bullet)$ for $(x, t) \in C$ and $t<t_{s}\left(y_{0}\right)$. We define $\left(x_{s}\left(y_{0}\right), t_{s}\left(y_{0}\right)\right)$ as a singularity point. Let $S$ be the set of all the singularity points.

We introduce a singularity mapping based on the properties of characteristics. Define a singularity mapping

$$
\mathscr{S}(y)=\left(x_{s}(y), t_{s}(y)\right)
$$

from a subset of $\mathbb{R}^{n}$ to $\mathbb{R}^{n} \times(0, \infty)$. We prove $t_{s}\left(y_{0}\right)$ is finite if and only if

$$
H\left(D g\left(y_{0}\right)\right) \neq \operatorname{conv} H\left(D g\left(y_{0}\right)\right) \text {, }
$$

where convH is convex hull of $H$,

$$
\operatorname{conv} H(x)=\inf \left\{\sum_{i=1}^{m} \lambda_{i} H\left(x_{i}\right) \mid \sum_{i=1}^{m} \lambda_{i} x_{i}=x, \sum_{i=1}^{m} \lambda_{i}=1, \lambda_{i} \geq 0, m=1,2, \cdots\right\} .
$$

Thus the domain of definition of $\mathscr{S}$ is

$$
\widetilde{\mathbb{R}}^{n}=\left\{y \in \mathbb{R}^{n} \mid H(D g(y)) \neq \operatorname{conv} H(D g(y))\right\}
$$

and

$$
S=\left\{\left(x_{s}(y), t_{s}(y)\right) \mid y \in \widetilde{\mathbb{R}}^{n}\right\} .
$$

Furthermore we prove the singularity mapping is continuous from $\widetilde{\mathbb{R}}^{n} \subset \mathbb{R}^{n}$ to $\mathbb{R}^{n} \times(0, \infty)$.

In the second part, we first investigate the differentiability of the solution. It will be proved that $u(x, t)$ is non-differentiable at $\left(x_{0}, t_{0}\right)$ if $G\left(x_{0}, t_{0}, \bullet\right)$ has more than one maximizing point and $\left(x_{0}, t_{0}\right)$ is a cluster point of non-differentiable points if $G\left(x_{0}, t_{0}, \bullet\right)$ has a unique degenerate maximizing point, which implies that $\left(x_{0}, t_{0}\right)$ is a singularity point of $u(x, t)$ if and only if $G\left(x_{0}, t_{0}, \bullet\right)$ has a unique degenerate maximizing point or has more than one maximizing point. Thus we can also call a point a singularity point if it is a non-differentiable point of the solution $u(x, t)$ or a cluster point of non-differentiable points of $u(x, t)$. We will show that the solution 
$u(x, t)$ is $C^{k}$ smooth in some neighborhood of $\left(x_{0}, t_{0}\right)$ if and only if there exists a unique non-degenerate maximizing point for $G\left(x_{0}, t_{0}, \bullet\right)$.

We are interested in the global structure of $S$. We will show that the set of singularity points consists of several path connected components. The set

$$
\left\{(D g(y), H(D g(y))) \mid y \in \mathbb{R}^{n}\right\} \backslash\left\{(D g(y), \operatorname{conv} H(D g(y))) \mid y \in \mathbb{R}^{n}\right\},
$$

denoted as

$$
\left(D g\left(\mathbb{R}^{n}\right), H\left(D g\left(\mathbb{R}^{n}\right)\right)\right) \backslash\left(D g\left(\mathbb{R}^{n}\right), \operatorname{conv} H\left(D g\left(\mathbb{R}^{n}\right)\right),\right.
$$

consists of path connected components of these hypersurfaces. We will show that there exists a one-to-one correspondence between the path connected components of the set of singularity points and the path connected components of the set $\left(D g\left(\mathbb{R}^{n}\right), \operatorname{conv} H\left(D g\left(\mathbb{R}^{n}\right)\right) \backslash\left(D g\left(\mathbb{R}^{n}\right), H\left(D g\left(\mathbb{R}^{n}\right)\right)\right)\right.$. Furthermore, each path connected component $S_{i}$ of the set of singularity points never vanishes as $t$ increases. In fact, these results depend only on $H$ and its domain of definition $D g\left(\mathbb{R}^{n}\right)$.

\section{Hopf's formula (II) and characteristics}

In this section we will give several lemmas on characteristics. Based on these lemmas we introduce a singularity mapping which plays an important role in studying the regularity and global structure of the HJ solutions.

Bardi and Evans showed that

$$
u(x, t)=\max _{p \in \Omega} \bar{G}(x, t, p),
$$

where

$$
\Omega=\left\{p \in \mathbb{R}^{n} \mid g^{*}(p)<\infty\right\} .
$$

Set $p=D g(y)$ in (2.1), we will show:

Lemma 2.1. $u(x, t)=\max _{y \in \mathbb{R}^{n}} G(x, t, y)$.

Proof. First we show that

$$
g^{*}(D g(y))=y \cdot D g(y)-g(y), \forall y \in \mathbb{R}^{n} .
$$

It follows from the convexity of $g(y)$ that

$$
q \cdot D g(y)-g(q) \leq y \cdot D g(y)-g(y), \forall(q, y) \in \mathbb{R}^{n} \times \mathbb{R}^{n},
$$

which implies for each $y \in \mathbb{R}^{n}$

$$
g^{*}(D g(y))=\sup _{q \in \mathbb{R}^{n}}\{q \cdot D g(y)-g(q)\}=y \cdot D g(y)-g(y) .
$$

Next we will show that

$$
D g\left(\mathbb{R}^{n}\right) \subset \Omega \subset \overline{D g\left(\mathbb{R}^{n}\right)}
$$


First we show $D g\left(\mathbb{R}^{n}\right) \subset \Omega$, which follows from (2.3).

Next we will prove $\overline{D g\left(\mathbb{R}^{n}\right)} c \Omega^{c}$, which is equivalent to $\Omega \subset \overline{D g\left(\mathbb{R}^{n}\right)}$.

For each $p \in \overline{D g\left(\mathbb{R}^{n}\right)}{ }^{c}$, we have

$$
d=d\left(p, \overline{D g\left(\mathbb{R}^{n}\right)}\right)=\inf _{q \in \mathbb{R}^{n}}|p-D g(q)|>0
$$

Consider a mapping $f$ from $\partial B(0, r)=\left\{q \in \mathbb{R}^{n}|| q \mid=r\right\}$ to $\partial B(0, r)$ as follows:

$$
q \longmapsto \frac{|q|(p-D g(q))}{|p-D g(q)|},
$$

where $q \in \partial B(0, r) . f$ is continuous according to $(2.7)$. Furthermore $f(\partial B(0, r)) \neq$ $\partial B(0, r)$ since $p \in{\overline{D g\left(\mathbb{R}^{n}\right)}}^{c}$ and $D g\left(\mathbb{R}^{n}\right)$ is convex and bounded.

Using fixed point theorem we have

$$
\begin{aligned}
& \forall r>0, \exists q_{r} \in \partial B(0, r)=\left\{q \in \mathbb{R}^{n}|| q \mid=r\right\} \text { such } \\
& \text { that } p-D g\left(q_{r}\right)=k q_{r}, \text { where } k=\left|p-D g\left(q_{r}\right)\right| /\left|q_{r}\right| .
\end{aligned}
$$

It follows from (2.7) and (2.8) that

$$
\left(p-D g\left(q_{r}\right)\right) \cdot q_{r}=\left|q_{r}\right|\left|p-D g\left(q_{r}\right)\right| \geq r d .
$$

Using the convexity of $g$ and (2.9), we have for each $r>0$ there exists a $q_{r} \in \partial B(0, r)$ such that $p \cdot q_{r}-g\left(q_{r}\right) \geq\left(p-D g\left(q_{r}\right)\right) \cdot q_{r}-g(0) \geq r d-g(0)$, Consequently,

$$
g^{*}(p)=\sup _{q \in \mathbb{R}^{n}}\{p \cdot q-g(q)\}=+\infty,
$$

which implies $p \in \Omega^{c}$. Then $\Omega \subset \overline{D g\left(\mathbb{R}^{n}\right)}$.

Using (2.3), we have

$$
\begin{aligned}
G(x, t, y) & =g(y)+(x-y) \cdot D g(y)-t H(D g(y)) \\
& =x \cdot D g(y)-g^{*}(D g(y))-t H(D g(y))
\end{aligned}
$$

Next we prove

$$
u(x, t)=\sup _{y \in \mathbb{R}^{n}} G(x, t, y) .
$$

Setting $q=0$ in (2.4), we have

$$
g(y)-y \cdot D g(y) \leq g(0)<+\infty,
$$

which implies that $\sup _{y \in \mathbb{R}^{n}} G(x, t, y)$ exists. Using (2.1), (2.6) and (2.10) gives

$$
\begin{aligned}
& \sup _{y \in \mathbb{R}^{n}} G(x, t, y)=\sup _{y \in \mathbb{R}^{n}}\left\{x \cdot D g(y)-g^{*}(D g(y))-t H(D g(y))\right\} \\
\leq & \max _{p \in \Omega} \bar{G}(x, t, p)=u(x, t) .
\end{aligned}
$$


On the other hand, there exists a maximizing point $p_{0} \in \Omega$ for $\bar{G}(x, t, \bullet)$. According to $(2.6)$, there exists a sequence $D g\left(y_{n}\right) \rightarrow p_{0}$ as $n \rightarrow \infty$. Noticing $g^{*}$ is continuous, we have

$$
\begin{aligned}
& u(x, t)=x \cdot p_{0}-g^{*}\left(p_{0}\right)-t H\left(p_{0}\right) \\
= & \lim _{n \rightarrow \infty}\left\{x \cdot D g\left(y_{n}\right)-g^{*}\left(D g\left(y_{n}\right)\right)-t H\left(D g\left(y_{n}\right)\right)\right\} \\
\leq & \sup _{y \in \mathbb{R}^{n}}\left\{x \cdot D g(y)-g^{*}(D g(y))-t H(D g(y))\right\} \\
= & \sup _{y \in \mathbb{R}^{n}} G(x, t, y) .
\end{aligned}
$$

Using (2.13) and (2.14), we have

$$
u(x, t)=\sup _{y \in \mathbb{R}^{n}}\left\{x \cdot D g(y)-g^{*}(D g(y))-t H(D g(y))\right\} .
$$

Thus (2.11) is proved. For each given point $(x, t)$, the domain of dependence of HJ equations is finite since it is hyperbolic type, thus the supremum in (2.15) is a maximum.

According to lemma 2.1, a maximizing point $y$ for $G(x, t, \bullet)$ is a critical point for $G(x, t, \bullet)$, i.e.,

$$
D_{y} G(x, t, y)=[x-y-t D H(D g(y))] \cdot D^{2} g(y)=0,
$$

which yields

$$
\frac{x-y}{t}=D H(D g(y))
$$

Moreover,

$$
\begin{aligned}
& D_{t} G(x, t, y)=-H(D g(y)), \\
& D_{x} G(x, t, y)=D g(y) .
\end{aligned}
$$

If $D_{y} G(x, t, y)=0$, then

$$
D_{y}^{2} G(x, t, y)=D^{2} g(y)\left[-I-t D^{2} H(D g(y)) \cdot D^{2} g(y)\right] .
$$

On the other hand, for $\bar{G}(x, t, p)$ defined by (1.6), we have

$$
D_{p} \bar{G}(x, t, p)=x-D g^{*}(p)-t D H(p),
$$

which gives

$$
D_{p} \bar{G}(x, t, p)=0 \Longleftrightarrow p=D g(x-t D H(p)) .
$$

Definition 2.1. Let $y_{0}$ be a maximizing point for $G\left(x_{0}, t_{0}, \bullet\right)$. Then $y_{0}$ is called nondegenerate (respectively, degenerate) if $\left|D_{y}^{2} G\left(x_{0}, t_{0}, y_{0}\right)\right| \neq 0($ respectively, $=0)$.

\section{Lemma 2.2. Let}

$$
U=\{(x, t) \mid \exists \text { unique non-degenerate maximizing point for } G(x, t, \bullet)\} \text {. }
$$


Then $U$ is an open subset of $\mathbb{R}^{n} \times(0, \infty)$, and $u(x, t)$ is $C^{k}$ smooth on $U$.

Proof. The proof is similar to that of Lemma 2.1 in [?].

Lemma 2.3. Suppose $\left(x_{0}, t_{0}\right) \in \mathbb{R}^{n} \times(0, \infty), y_{0}=y\left(x_{0}, t_{0}\right)$ is a point which maximizes $G\left(x_{0}, t_{0}, \bullet\right), l$ is an open straightline segment joining $\left(x_{0}, t_{0}\right)$ to $\left(y_{0}, 0\right)$, and $\left(x_{1}, t_{1}\right)$ is any point belonging to $l$. Then there is a unique point $y\left(x_{1}, t_{1}\right)=$ $y\left(x_{0}, t_{0}\right)=y_{0}$ which maximizes $G\left(x_{1}, t_{1}, \bullet\right)$.

Proof. Assume

$$
G\left(x_{1}, t_{1}, y_{1}\right)=\max _{y \in \mathbb{R}^{n}} G\left(x_{1}, t_{1}, y\right),
$$

where $\left(x_{1}, t_{1}\right) \in l$. Suppose $y_{1} \neq y_{0}$. (2.24) implies that

$$
G\left(x_{1}, t_{1}, y_{1}\right) \geq G\left(x_{1}, t_{1}, y_{0}\right),
$$

i.e.,

$$
\begin{aligned}
& \frac{1}{t_{1}} g\left(y_{1}\right)+\frac{x_{1}-y_{1}}{t_{1}} D g\left(y_{1}\right)-H\left(D g\left(y_{1}\right)\right) \\
\geq & \frac{1}{t_{1}} g\left(y_{0}\right)+\frac{x_{1}-y_{0}}{t_{1}} D g\left(y_{0}\right)-H\left(D g\left(y_{0}\right)\right) .
\end{aligned}
$$

This, together with the observation $\left(x_{1}-y_{0}\right) / t_{1}=\left(x_{0}-y_{0}\right) / t_{0}$, gives

$$
\begin{aligned}
& \frac{1}{t_{1}} g\left(y_{1}\right)+\left(\frac{1}{t_{0}}-\frac{1}{t_{1}}\right) g\left(y_{0}\right)+\frac{x_{1}-y_{1}}{t_{1}} D g\left(y_{1}\right)-H\left(D g\left(y_{1}\right)\right) \\
\geq & \frac{1}{t_{0}} G\left(x_{0}, t_{0}, y_{0}\right) .
\end{aligned}
$$

Since $g(y)$ is strictly convex, we have

$$
g\left(y_{0}\right)-g\left(y_{1}\right)-\left(y_{0}-y_{1}\right) \cdot D g\left(y_{1}\right)>0,
$$

which gives

$$
\left(\frac{1}{t_{0}}-\frac{1}{t_{1}}\right)\left[g\left(y_{1}\right)-g\left(y_{0}\right)\right]+\left(\frac{1}{t_{0}}-\frac{1}{t_{1}}\right)\left(y_{0}-y_{1}\right) \cdot D g\left(y_{1}\right)>0 .
$$

It can be verified (using again $\left.\left(x_{1}-y_{0}\right) / t_{1}=\left(x_{0}-y_{0}\right) / t_{0}\right)$ that

$$
\frac{x_{0}-y_{1}}{t_{0}}-\frac{x_{1}-y_{1}}{t_{1}}=\left(\frac{1}{t_{0}}-\frac{1}{t_{1}}\right)\left(y_{0}-y_{1}\right) \text {. }
$$

Then according to (2.27), (2.29) and (2.30) we have

$$
\begin{aligned}
& \frac{1}{t_{0}} G\left(x_{0}, t_{0}, y_{0}\right) \\
\leq & \frac{1}{t_{1}} g\left(y_{1}\right)+\left(\frac{1}{t_{0}}-\frac{1}{t_{1}}\right) g\left(y_{0}\right)+\frac{x_{1}-y_{1}}{t_{1}} D g\left(y_{1}\right)-H\left(D g\left(y_{1}\right)\right) \\
< & \frac{1}{t_{0}} g\left(y_{1}\right)+\frac{x_{0}-y_{1}}{t_{0}} D g\left(y_{1}\right)-H\left(D g\left(y_{1}\right)\right) \\
= & \frac{1}{t_{0}} G\left(x_{0}, t_{0}, y_{1}\right),
\end{aligned}
$$


which contradicts to the fact that $y_{0}$ is a maximizing point for $G\left(x_{0}, t_{0}, \bullet\right)$.

Lemma 2.4. Let $y_{0} \in \mathbb{R}^{n}$ and assume the corresponding characteristic $C$ is defined by (1.10). If $g \in C^{k}$ satisfies (1.2) and (1.3), then precisely one of the following statements must hold:

(i) $y_{0}$ is the unique non-degenerate maximizing point for $G(x, t, \bullet)$ for each $(x, t) \in$ $C$; or

(ii) there exits a point $\left(x_{s}\left(y_{0}\right), t_{s}\left(y_{0}\right)\right) \in C$ such that $y_{0}$ is either the unique degenerate maximizing point for $G\left(x_{s}\left(y_{0}\right), t_{s}\left(y_{0}\right), \bullet\right)$ or one of the maximizing points for $G\left(x_{s}\left(y_{0}\right), t_{s}\left(y_{0}\right), \bullet\right)$. Furthermore, $y_{0}$ is the unique non-degenerate maximizing point for $G(x, t, \bullet)$ for each $(x, t) \in C^{-}=C \cap\left\{(x, t) \mid t_{s}\left(y_{0}\right)>t>0\right\}$; while for $(x, t) \in C^{+}=C \cap\left\{(x, t) \mid t>t_{s}\left(y_{0}\right)\right\}, y_{0}$ is no longer the maximizing point for $G(x, t, \bullet)$.

Proof. First we prove that $y_{0}$ is no longer a maximizing point for $G(x, t, \bullet)$ for $(x, t) \in C^{+}$if there exists more than one maximizing point for $G\left(x_{s}\left(y_{0}\right), t_{s}\left(y_{0}\right), \bullet\right)$. If this is not true, then there exists a point $(\tilde{x}, \tilde{t}) \in C^{+}$such that $y_{0}$ is a maximizing point for $G(\tilde{x}, \tilde{t}, \bullet)$. Consequently, $y_{0}$ is the unique maximizing point for $G\left(x_{s}\left(y_{0}\right), t_{s}\left(y_{0}\right), \bullet\right)$ according to Lemma 2.3 , which is a contradiction since there are more than one maximizing point for $G\left(x_{s}\left(y_{0}\right), t_{s}\left(y_{0}\right), \bullet\right)$.

If $y_{0}$ is a unique degenerate maximizing point for $G\left(x_{s}\left(y_{0}\right), t_{s}\left(y_{0}\right)\right.$, •), i.e.,

$$
\left|D_{y}^{2} G\left(x_{s}\left(y_{0}\right), t_{s}\left(y_{0}\right), y_{0}\right)\right|=0 .
$$

From (2.32), there exists a non-zero vector $\xi \in \mathbb{R}^{n}$ such that

$$
\xi^{T} D_{y}^{2} G\left(x_{s}\left(y_{0}\right), t_{s}\left(y_{0}\right), y_{0}\right) \xi=0,
$$

i.e.,

$$
-\xi^{T} D^{2} g\left(y_{0}\right) \xi-t_{s}\left(y_{0}\right) \xi^{T} D^{2} g\left(y_{0}\right) D^{2} H\left(D g\left(y_{0}\right)\right) D^{2} g\left(y_{0}\right) \xi=0 .
$$

Introduce the function

$$
\bar{g}(t)=-\xi^{T} D^{2} g\left(y_{0}\right) \xi-t \xi^{T} D^{2} g\left(y_{0}\right) D^{2} H\left(D g\left(y_{0}\right)\right) D^{2} g\left(y_{0}\right) \xi .
$$

Then

$$
\xi^{T} D_{y}^{2} G\left(x, t, y_{0}\right) \xi=\bar{g}(t) .
$$

According to Lemma 2.3, $y_{0}$ is a unique maximizing point for $G(x, t, \bullet)$ for each $(x, t) \in C$ and $t \leq t_{s}\left(y_{0}\right)$, since $y_{0}$ is a maximizing point for $G\left(x_{s}\left(y_{0}\right), t_{s}\left(y_{0}\right), \bullet\right)$. Then

$$
\xi^{T} D_{y}^{2} G\left(x, t, y_{0}\right) \xi \leq 0, \text { for }(x, t) \in C, t \leq t_{s}\left(y_{0}\right) .
$$

On the other hand, (2.34) is a linear function of $t$ and has a unique zero point, $t=t_{s}\left(y_{0}\right)$. Thus

$$
\bar{g}(t)>0, \text { for } t>t_{s}\left(y_{0}\right) .
$$


It follows from (2.35) and (2.37) that

$$
\xi^{T} D_{y}^{2} G\left(x, t, y_{0}\right) \xi>0, \text { for }(x, t) \in C, t>t_{s}\left(y_{0}\right),
$$

which implies that the matrix $D_{y}^{2} G\left(x, t, y_{0}\right)$ is positive definite or non-definite. Then $y_{0}$ cannot be a maximizing point for $G(x, t, \bullet)$ for $(x, t) \in C$ and $t>t_{s}\left(y_{0}\right)$. The proof is complete.

The above lemma was obtained by Li and Wang [8] for convex scalar conservation laws.

From the above lemma, we see the supremum of $T$ in (1.11) is indeed a maximum when the supremum is finite. Consequently, for each $y_{0} \in \mathbb{R}^{n}$ satisfying $t_{s}\left(y_{0}\right)<\infty$, we see

$$
\left\{\begin{array}{l}
t_{s}\left(y_{0}\right)=\max \left\{0<t<\infty \mid u(x, t)=G\left(x, t, y_{0}\right),(x, t) \in C\right\}, \\
x_{s}\left(y_{0}\right)=y_{0}+t_{s}\left(y_{0}\right) D H\left(D g\left(y_{0}\right)\right),
\end{array}\right.
$$

where the characteristic $C$ is defined by (1.10). We define the point $\left(x_{s}\left(y_{0}\right), t_{s}\left(y_{0}\right)\right)$ as singularity point of solution $u(x, t)$ and let $S$ be the set of singularity points. In order to study the structure of the set of singularity points we introduce a singularity mapping $\mathscr{S}$ from some subset of $\mathbb{R}^{n}$ to $\mathbb{R}^{n} \times(0, \infty)$,

$$
\mathscr{S}\left(y_{0}\right)=\left(x_{s}\left(y_{0}\right), t_{s}\left(y_{0}\right)\right) .
$$

In other words, $\left(x_{s}\left(y_{0}\right), t_{s}\left(y_{0}\right)\right)$ is the point such that $G\left(x_{s}\left(y_{0}\right), t_{s}\left(y_{0}\right), \bullet\right)$ has a unique degenerate maximizing point or more than one maximizing point. Similar to the proof of Lemma 2.4 of [?], we have the following result.

Lemma 2.5. $\mathscr{S}$ defined by (2.40) is a continuous map.

Lemma 2.6. Let $C$ be defined by (1.10). Then $y_{0}$ is a unique non-degenerate maximizing point for $G(x, t, \bullet)$ for $(x, t) \in C$ if and only if $H\left(D g\left(y_{0}\right)\right)=\operatorname{convH}\left(D g\left(y_{0}\right)\right)$, where convH is convex hull of $H$, conv $H(x)$ is defined by (1.14).

Proof. For each $y \neq y_{0}$, using (1.5) gives

$$
\begin{aligned}
& G\left(x, t, y_{0}\right)-G(x, t, y) \\
= & g\left(y_{0}\right)+\left(x-y_{0}\right) \cdot D g\left(y_{0}\right)-t H\left(D g\left(y_{0}\right)\right)-g(y)-(x-y) \cdot D g(y)+t H(D g(y)) \\
= & g\left(y_{0}\right)-g(y)-D g(y) \cdot\left(y_{0}-y\right) \\
& +t\left(H(D g(y))-H\left(D g\left(y_{0}\right)\right)\right)-\left(x-y_{0}\right) \cdot\left(D g(y)-D g\left(y_{0}\right)\right) \\
= & g\left(y_{0}\right)-g(y)-D g(y) \cdot\left(y_{0}-y\right) \\
& \left.+t\left[H(D g(y))-H\left(D g\left(y_{0}\right)\right)\right)-D H\left(D g\left(y_{0}\right)\right) \cdot\left(D g(y)-D g\left(y_{0}\right)\right)\right],
\end{aligned}
$$

where we have used the fact that $x=y_{0}+t D H\left(D g\left(y_{0}\right)\right)$.

Necessary condition: assume $y_{0}$ is a unique maximizing point for $G(x, t, \bullet)$ for each $(x, t) \in C$ implies $G\left(x, t, y_{0}\right)-G(x, t, y)>0$ for each $(x, t) \in C$ and each $y \neq y_{0}$. Dividing (2.41) by $t$ and letting $t \rightarrow \infty$ yield

$$
H(D g(y))-H\left(D g\left(y_{0}\right)\right)-D H\left(D g\left(y_{0}\right)\right) \cdot\left(D g(y)-D g\left(y_{0}\right)\right) \geq 0
$$


for $y \neq y_{0}$. Thus convH$\left(D g\left(y_{0}\right)\right)=H\left(D g\left(y_{0}\right)\right)$.

Sufficient condition: assume $H\left(D g\left(y_{0}\right)\right)=\operatorname{conv} H\left(D g\left(y_{0}\right)\right)$. This implies that (2.42) holds. Since $D^{2} g(y)>0$, using (2.41) and (2.42) gives

$$
G\left(x, t, y_{0}\right)-G(x, t, y)>0, \quad \text { for }(x, t) \in C \text { and } y \neq y_{0},
$$

which implies that $y_{0}$ is a unique non-degenerate maximizing point for $G(x, t, \bullet)$ for $(x, t) \in C$. Otherwise there exists a point $\left(x_{1}, t_{1}\right) \in C$ such that $y_{0}$ is a degenerate maximizing point for $G\left(x_{1}, t_{1}, \bullet\right)$. Then $y_{0}$ is no longer a maximizing point for $G(x, t, \bullet)$ for $(x, t) \in C$ and $t>t_{1}$ according to Lemma 2.4. This is a contradiction

Kruzhkov and Petrosyan in [7] obtained a similar result for scalar conservation laws of one space dimension with nondecreasing initial data and for the HamiltonJacobi equation of one space dimension with convex initial data.

Remark 2.1. The assumption 1.4 that $D g\left(\mathbb{R}^{n}\right)$ is convex is a necessary condition such that there exists convex hull of $H$ when $H$ is defined on $D g\left(\mathbb{R}^{n}\right)$.

From Lemma 2.6 the domain of definition of the singularity mapping $\mathscr{S}$ is $\widetilde{\mathbb{R}}^{n}$, where

$$
\widetilde{\mathbb{R}}^{n}=\left\{y \in \mathbb{R}^{n} \mid H(D g(y)) \neq \operatorname{conv} H(D g(y))\right\} .
$$

Then the singularity mapping $\mathscr{S}$ is continuous from $\widetilde{\mathbb{R}}^{n}$ to $\mathbb{R}^{n} \times(0, \infty)$ and the set of singularity points formed by all singularity points defined by (2.39) can be written in the following form:

$$
S=\left\{\left(x_{s}(y), t_{s}(y)\right) \mid x_{s}(y)=y+t_{s}(y) D H(D g(y)), y \in \widetilde{\mathbb{R}}^{n}\right\},
$$

where

$$
\begin{aligned}
t_{s}(y) & =\max \{0<t<\infty \mid u(x, t)=G(x, t, y),(x, t) \in C\}, \\
x_{s}(y) & =y+t_{s}(y) D H(D g(y)), \\
C & =\{(x, t) \mid x=y+t D H(D g(y))\} .
\end{aligned}
$$

\section{Regularity and global structure of solution}

In this section we are mainly concerned with the global structure of the set of singularity points $S$ of the solution $u(x, t)$ in the upper half space $\mathbb{R}^{n} \times(0, \infty)$. We will show that $S$, as the complementary set of the set $U$ in lemma 2.2 , is a closure of the set consisting of points at which solution is non-differentiable. Then as a corollary the solution $u(x, t)$ is $C^{k}$ smooth in some neighborhood of $\left(x_{0}, t_{0}\right)$ if and only if there is a unique non-degenerate maximizing point for $G\left(x_{0}, t_{0}, \bullet\right)$. The set of singularity points consists of several path connected components $S_{i}$. We will show that there exists a one-to-one correspondence between the path connected components $S_{i}$ of the set of singularity points and path connected components of $\left(D g\left(\mathbb{R}^{n}\right), H\left(D g\left(\mathbb{R}^{n}\right)\right)\right)$ 
$\backslash\left(D g\left(\mathbb{R}^{n}\right)\right.$, convH $\left.\left(D g\left(\mathbb{R}^{n}\right)\right)\right)$. Furthermore, each path connected component $S_{i}$ of the set of singularity points never vanishes as $t$ increases. Our results depend only on the Hamiltonian $H$ and its domain of definition $D g\left(\mathbb{R}^{n}\right)$.

Lemma 3.1. If $G\left(x_{0}, t_{0}, \bullet\right)$ has a unique degenerate maximizing point or more than one maximizing point, then $u(x, t)$ is not differentiable in any neighborhood $U_{\left(x_{0}, t_{0}\right)}$ of $\left(x_{0}, t_{0}\right)$.

The proof is similar to that of Lemma 3.1 in [?]. Here, we outline the sketch proof of it: it follows from Theorem 2.1 of Hoang [6] that $(x, t)$ is a non-differentiable point of the solution $u(x, t)$ if $G(x, t, \bullet)$ has more than one maximizing point; there exists a non-differentiable point of the solution $u(x, t)$ in any neighborhood of $\left(x_{0}, t_{0}\right)$ if $G(x, t, \bullet)$ has a unique degenerate maximizing point.

Let

$S^{1}=\left\{(x, t) \in \mathbb{R}^{n} \times(0, \infty) \mid G(x, t, \bullet)\right.$ has a unique degenerate maximizing point $\}$,

$S^{2}=\left\{(x, t) \in \mathbb{R}^{n} \times(0, \infty) \mid G(x, t, \bullet)\right.$ has more than one maximizing point $\}$.

From the above proof, we see that each point of $S^{1}$ is a cluster point of points of $S^{2}$. Furthermore $S$ as the set of singularity points is a closure of $S^{2}$. Then

$$
S=S^{1} \bigcup S^{2}=\left\{\left(x_{s}(y), t_{s}(y)\right) \mid y \in \widetilde{\mathbb{R}}^{n}\right\} .
$$

Thus an equivalent definition of a singularity point can be given: a point is called a singularity point if it is a non-differentiable point of the solution $u(x, t)$ or a cluster point of non-differentiable points of the solution $u(x, t)$. Therefore as a corollary of lemma 2.2 and lemma 3.1 we have the following result.

Theorem 3.1. The solution $u(x, t)$ is $C^{k}$ smooth in some neighborhood of $\left(x_{0}, t_{0}\right)$ if and only if there is a unique non-degenerate maximizing point for $G\left(x_{0}, t_{0}, \bullet\right)$.

We see the set $\widetilde{\mathbb{R}}^{n}=\left\{y \in \mathbb{R}^{n} \mid H(D g(y)) \neq \operatorname{convH}(D g(y))\right\}$ is an open subset of $\mathbb{R}^{n}$ since the functions $H(D g(\bullet))$ and $\operatorname{conv} H(D g(\bullet))$ are continuous on $\mathbb{R}^{n}$. Thus $\widetilde{\mathbb{R}}^{n}$ is union of path connected components $R_{i}$, i.e.,

$$
\widetilde{\mathbb{R}}^{n}=\bigcup R_{i}
$$

Let

$$
J=\left(D g\left(\mathbb{R}^{n}\right), H\left(D g\left(\mathbb{R}^{n}\right)\right)\right) \backslash\left(D g\left(\mathbb{R}^{n}\right), \operatorname{conv} H\left(D g\left(\mathbb{R}^{n}\right)\right)\right)=\bigcup J_{i},
$$

where

$$
J_{i}=\left(D g\left(R_{i}\right), H\left(D g\left(R_{i}\right)\right)\right)=\left\{(D g(y), H(D g(y))) \mid y \in R_{i}\right\}
$$

is a path connected component of $J$. Then we have

Theorem 3.2. $S_{i}=\mathscr{S}\left(R_{i}\right)$ is a path connected component of the set of singularity points, which never vanishes for $t>t_{i}$, where $t_{i}$ is the formation time of $S_{i}$ and 
$S=\bigcup S_{i}$. Furthermore there exists a one-to-one correspondence between the path connected components of the set of singularity points and path connected components of J. More precisely, there exists a one-to-one correspondence between the set $S_{i}$ and $J_{i}$.

Proof. First we claim that

there exists a one-to-one correspondence between $S_{i}$ and $R_{i}$.

It follows from Lemma 2.6 that

$$
\begin{aligned}
& y \text { is the unique non-degenerate maximizing point for } G(x, t, \bullet) \\
& \text { for each }(x, t) \in\left\{(x, t) \mid x=y+t D H(D g(y)), y \in \partial R_{i}\right\} .
\end{aligned}
$$

Thus the characteristic emanating from $\partial R_{i}$ will not intersect with each other, which implies the mapping $y \longmapsto(x, T)$ is one-to-one and continuous, where $x=$ $y+T D H(D g(y)), y \in \partial R_{i}, T>0$ is fixed. Let

$$
\partial \Pi_{i}=\bigcup\left\{(x, t) \mid x=y+t D H(D g(y)), y \in \partial R_{i}, t>0\right\},
$$

i.e., $\partial \Pi_{i}$ is composed of valid characteristics emanating from $\partial R_{i}$ according to (3.8). Let

$$
\Pi_{i}=\bigcup\left\{(x, t) \mid x=y+t D H(D g(y)), y \in R_{i}, 0<t \leq t_{s}(y)\right\},
$$

i.e., $\Pi_{i}$ is composed of all valid characteristic segment emanating from $R_{i}$.

Note that each characteristic emanating from $\partial R_{i}$ is valid and any other valid characteristic segment will not intersect with it. We can show that a valid characteristic segment from $R_{i}$ and a valid one from $R_{j},(i \neq j)$, can not intersect with each other, i.e.,

$$
\Pi_{i} \bigcap \Pi_{j}=\emptyset, i \neq j,
$$

(see Theorem 3.1 in [?] for a detailed proof). $\left(x_{s}(y), t_{s}(y)\right) \in \Pi_{i}, y \in R_{i}$. This implies $S_{i}=\mathscr{S}\left(R_{i}\right) \subset \Pi_{i}$.

For each $y \in \widetilde{\mathbb{R}}^{n}$, it is known that $\mathscr{S}(y)=\left(x_{s}(y), t_{s}(y)\right)$, where $x_{s}(y)$ and $t_{s}(y)$ are given by (2.46). Furthermore,

$$
\begin{aligned}
\mathscr{S}\left(\widetilde{\mathbb{R}}^{n}\right) & =\left\{\left(x_{s}(y), t_{s}(y)\right) \mid x_{s}(y)=y+t_{s}(y) D H(D g(y)), y \in \widetilde{\mathbb{R}}^{n}\right\} \\
& =\bigcup \mathscr{S}\left(R_{i}\right)=\bigcup S_{i}=S,
\end{aligned}
$$

where $S_{i}=\mathscr{S}\left(R_{i}\right)$. We have

$$
S_{i} \bigcap S_{j} \subset \Pi_{i} \bigcap \Pi_{j}=\emptyset(i \neq j) .
$$

Thus, $S_{i}=\mathscr{S}\left(R_{i}\right)$ is a path connected component of the set of the singularity points since the singularity mapping $\mathscr{S}$ is continuous and $R_{i}$ is path connected. Thus we have proved assertion (3.7). 
It follows from Lemma 2.6, the definition of $\widetilde{\mathbb{R}}^{n}$ and (3.4) that

$$
\begin{aligned}
J & =\left\{\left(D g\left(\mathbb{R}^{n}\right), H\left(D g\left(\mathbb{R}^{n}\right)\right)\right)\right\} \backslash\left\{\left(D g\left(\mathbb{R}^{n}\right), \operatorname{conv} H\left(D g\left(\mathbb{R}^{n}\right)\right)\right\}\right. \\
& =\left(D g\left(\widetilde{\mathbb{R}}^{n}\right), H\left(D g\left(\widetilde{\mathbb{R}}^{n}\right)\right)\right) \\
& =\left(D g\left(\bigcup R_{i}\right), H\left(D g\left(\bigcup R_{i}\right)\right)\right) \\
& =\left(\bigcup D g\left(R_{i}\right), \bigcup H\left(D g\left(R_{i}\right)\right)\right) \\
& =\bigcup\left(D g\left(R_{i}\right), H\left(D g\left(R_{i}\right)\right)\right)=\bigcup J_{i},
\end{aligned}
$$

where $J_{i}$ is defined in (3.6).

Now we claim

$$
J_{i} \text { is path connected component of } J \text {. }
$$

In fact $R_{i}$ is path connected component of $\widetilde{\mathbb{R}}^{n}$, which implies $R_{i} \bigcap R_{j}=\emptyset$ for $i \neq j$ and the mapping $H(D g(\bullet))$ is continuous. Consequently, $J_{i}$ is also path connected. Furthermore,

$$
J_{i} \bigcap J_{j}=\left(D g\left(R_{i}\right), H\left(D g\left(R_{i}\right)\right)\right) \bigcap\left(D g\left(R_{j}\right), H\left(D g\left(R_{j}\right)\right)\right)=\emptyset,
$$

since $D g$ is one to one from $\mathbb{R}^{n}$ to $D g\left(\mathbb{R}^{n}\right)$ and $R_{i} \bigcap R_{j}=\emptyset$ for $i \neq j$. So $J_{i}$ is a path connected component of $J$.

Based on (3.7), (3.13) and the fact that $D g$ is one to one from $\mathbb{R}^{n}$ to $D g\left(\mathbb{R}^{n}\right)$, we build a one-to-one correspondence between the following sets:

$$
S_{i} \stackrel{\mathscr{S}}{\longleftrightarrow} R_{i} \stackrel{D g}{\longleftrightarrow} D g\left(R_{i}\right) \stackrel{H}{\longleftrightarrow}\left(D g\left(R_{i}\right), H\left(D g\left(R_{i}\right)\right)\right) .
$$

Thus there exists a one-to-one correspondence between $S_{i}$ and $J_{i}$.

Finally, we will show that each path connected component $S_{i}$ never vanishes as $t$ increases.

Let $y_{0} \in \partial R_{i}$. Thus $t_{s}\left(y_{0}\right)=\infty$. Let $y_{n} \rightarrow y_{0}, y_{n} \in R_{i}$. We will show that $t_{s}\left(y_{n}\right) \rightarrow \infty$.

Let $T>0$ be arbitrarily large and $C_{n}: x=y_{n}+t D H\left(D g\left(y_{n}\right)\right), t \geq 0, n>0$. There exists a neighborhood $U_{\left(x_{T}, T\right)}$ of $\left(x_{T}, T\right) \in C$ (defined by $\left.(1.10)\right)$ such that there exists a unique non-degenerate maximizing point for $G(x, t, \bullet)$ for each $(x, t) \in$ $U_{\left(x_{T}, T\right)}$. On the other hand, there exists $N>0$ such that $C_{n}$ passes through $U_{\left(x_{T}, T\right)}$ if $n>N$ since $y_{n} \rightarrow y_{0}$. Thus $t_{s}\left(y_{n}\right)>T$, which implies $t_{s}\left(y_{n}\right) \rightarrow \infty$.

Consider a point $y_{0} \in \partial R_{i}$, then

there exists a point $y \in R_{i} \bigcap U_{y_{0}}$ such that

$t_{s}(y)<\infty$ for each neighborhood $U_{y_{0}}$ of $y_{0}$.

Let $\left(x_{s}\left(y_{n}\right), t_{s}\left(y_{n}\right)\right) \in S_{i}$ with $R_{i} \ni y_{n} \rightarrow y_{0}$. Thus $t_{s}\left(y_{n}\right) \rightarrow \infty$, which implies that $S_{i}$ will never vanish since $S_{i}$ is path connected. This completes the proof.

The above results depend only on the Hamiltonian $H$ and its domain of definition $D g\left(\mathbb{R}^{n}\right)$. 
Using Theorem 3.2 we have the following corollaries.

Corollary 3.1. The domain of dependence of a point $(x, t) \in C:\{(x, t) \mid x=$ $\left.y+t D H(D g(y)), y \in \mathbb{R}^{n} \backslash \widetilde{\mathbb{R}}^{n}\right\}$ is the point $y$. The domain of influence of point $y \in \mathbb{R}^{n} \backslash \widetilde{\mathbb{R}}^{n}$ is $C$.

Corollary 3.2. The domain of dependence of a point $(x, t) \in \Pi_{i}$ is $R_{i} \cap B\left(x, M_{1} t\right)$, where $M_{1}=\sup _{y \in \mathbb{R}^{n}}|D H(D g(y))|$. The domain of influence of a point $y \in R_{i}$ is

$$
\Pi_{i} \bigcap\left\{(y+\xi t, t)|| \xi \mid \leq M_{1}\right\}
$$

\section{Concluding Remarks}

In the following two propositions, we have improved propositions 2.7 and 2.8 in [15]. Consequently, we have also gotten the same results of the paper mentioned above under a weaker assumption on initial data.

Proposition 4.1. Assume that $\sup _{y \in \mathbb{R}^{n}}|g(y)|<\infty, g(y)$ does not attain its minimum at $y_{0}$ and $D g\left(y_{0}\right) \neq 0$. Let $C=\left\{(x, t) \mid x=y_{0}+t D H\left(D g\left(y_{0}\right)\right), t>0\right\}$. Then there exists $(\tilde{x}, \tilde{t}) \in C$ such that $D g\left(y_{0}\right)$ is not a minimizing point for $F(\tilde{x}, \tilde{t}, \bullet)$.

Proof. Set

$$
y_{n}=y_{0}+t_{n}\left(D H\left(D g\left(y_{0}\right)\right)-D H(0)\right)
$$

thus

$$
x_{n}=y_{0}+t_{n} D H\left(D g\left(y_{0}\right)\right)=y_{n}+t_{n} D H(0),
$$

where $\left(x_{n}, t_{n}\right) \in C$.

$$
\begin{aligned}
& F\left(x_{n}, t_{n}, D g\left(y_{0}\right)\right)-F\left(x_{n}, t_{n}, 0\right) \\
= & g\left(x_{n}-t_{n} D H\left(D g\left(y_{0}\right)\right)\right)-g\left(x_{n}-t_{n} D H(0)\right)+t_{n}\left(L\left(D H\left(D g\left(y_{0}\right)\right)\right)-L(D H(0))\right) \\
= & g\left(y_{0}\right)-g\left(y_{n}\right)+t_{n}\left(L\left(D H\left(D g\left(y_{0}\right)\right)\right)-L(D H(0))\right) \\
> & 0
\end{aligned}
$$

for $t_{n}$ big enough since $g$ is bounded and $L\left(D H\left(D g\left(y_{0}\right)\right)\right)-L(D H(0)>0$.

It is worth pointing out that the conclusion of proposition 4.1 is the same to proposition 2.7 in [15] while the condition that $D g(y) \rightarrow 0$ as $|y| \rightarrow \infty$ of proposition 2.7 in [15], is not required.

Proposition 4.2. Assume that $g(y)$ does not attain its minimum at $y_{0}$ and $D g\left(y_{0}\right)=0$. Let $C=\left\{(x, t) \mid x=y_{0}+t D H\left(D g\left(y_{0}\right)\right), t>0\right\}$. Then there exists $(\tilde{x}, \tilde{t}) \in C$ such that $D g\left(y_{0}\right)$ is not a minimizing point for $F(\tilde{x}, \tilde{t}, \bullet)$

\section{Proof.}


There exists a point $y_{1} \in \mathbb{R}^{n}$ such that $g\left(y_{0}\right)>g\left(y_{1}\right)$ since $g(y)$ does not attain its minimum at $y_{0}$.

For $(x, t) \in C$, set $x-t D H(p)=y_{1}$, thus

$$
\begin{aligned}
D H(p) & =\frac{x-y_{1}}{t} \\
& =\frac{y_{0}+t D H\left(D g\left(y_{0}\right)\right)-y_{1}}{t} \\
& =D H(0)+\frac{y_{0}-y_{1}}{t} .
\end{aligned}
$$

We have

$$
D^{2} H \geq \alpha I, D^{2} L \leq \frac{1}{\alpha} I
$$

since $D^{2} L(D H(p)) D^{2} H(p)=I$.

Note that $D g\left(y_{0}\right)=0,(4.2)$ and (4.3), we get

$$
\begin{aligned}
& F\left(x, t, D g\left(x_{0}\right)\right)-F(x, t, p) \\
= & g\left(y_{0}\right)-g\left(y_{1}\right)+t\left(L\left(D H(0)-L\left(D H(0)+\frac{y_{0}-y_{1}}{t}\right)\right)\right) \\
= & g\left(y_{0}\right)-g\left(y_{1}\right)-\frac{1}{t} \int_{0}^{1}(1-s)\left(y_{0}-y_{1}\right)^{T} D^{2} L\left(D H(0)+s \frac{y_{0}-y_{1}}{t}\right)\left(y_{0}-y_{1}\right) d s \\
\geq & g\left(y_{0}\right)-g\left(y_{1}\right)-\frac{1}{2 \alpha} \frac{\left|y_{0}-y_{1}\right|^{2}}{t} \\
> & 0
\end{aligned}
$$

for $(x, t) \in C, t$ big enough. The proof is then completed.

Consequently, we have the following theorem whose conclusion is the same to theorem 3.3 in [15], without the assumption that $D g(y) \rightarrow 0$ as $|y| \rightarrow \infty$

Theorem 4.1. Assume $g \in C^{k}$ satisfies $\sup _{y \in \mathbb{R}^{n}}|g(y)|<\infty$, and $\sup _{y \in \mathbb{R}^{n}}|D g(y)|<\infty$. Let $R_{i}$ be the path connected component of $\widetilde{\mathbb{R}}^{n}$ on which initial function does not attain its minimum. Then $S_{i}=\mathscr{S}\left(R_{i}\right)$ is a path connected component of the set of singularity points $S$ and never vanishes for $t>t_{i}$, where $t_{i}$ is the formation time of $S_{i}$. Moreover, there exists one-to-one correspondence between $S_{i}$ and $R_{i}$, and $S=\bigcup S_{i}$.

\section{Acknowledgments}

The first author was supported by China Postdoctoral Science Foundation under contract 148028. He also thanks Professor Pingwen Zhang for support and encouragements. The second author was partially supported by CERG Grants of Hong Kong Research Grant Council, FRG grants of Hong Kong Baptist University, and International Research Team of Complex Systems of Chinese Academy of Sciences. The third author was supported by National Natural Foundation of China under contract 10671116. 


\section{References}

[1] E.n.Barron, P.Cannasa, R.Jensen, C.Sinestrari, Regularity of HamiltonJacobi equations when for ward is backward, Indiana. Univ. Math. J., 48 (1999), 395-409.

[2] M. BARDi And L.C. Evans, On Hopf's Formulas for solutions of Hamilton-Jacobi equations, Nonlinear Anal., Theory, Methods \& Applications, 8 (1984), 1373-1381.

[3] E. Conway And E. Hopf, Hamilton's theory and generalized solutions of the Hamilton-Jacobi equation, J. Math. Mech., 13 (1964), 939-986.

[4] M.G. Crandall and P.L. Lions, Viscosity solutions of Hamilton-Jacobi equations, Trans. Am. Math. Soc, 277 (1983), 1-42.

[5] E. Hopf, Generalized solutions of nonlinear equations of first order, J. Math.Mech., 14 (1965) 951-973.

[6] N. HoAnG, The regularity of generalized solutions of Hamilton-Jacobi equations, Nonlinear Anal. 59 (2004) 745-757

[7] S.N. Kruzhkov and N.S. Petrosyan, Asymptotic behaviour of solutions of the Cauchy problem for first-order nonlinear equations, Usp. Math. Nauk, 42 (1987), 3-40 (in Russian).

[8] B. Li AND J. WANG, The global qualitative study of solutions to a conservation law (I), Sci. Special Math. Issue (1979), 12-24 (in Chinese).

[9] C.-T. Lin, E.TADmor $L^{1}$ Stability and error estimates for approximate HamiltonJacobi solutions, Numer.Math., 87: 701-735 (2001)

[10] P L Lions, Generalized Solutions of Hamilton-Jacobi Equations, Pitman Advance Publishing Program, 1982.

[11] D.G. Schaeffer, A regularity theorem for conservation laws, Adv. Math. 11 (1973), 368-386

[12] T.D. VAn, N. HoAng, N.D. Thai Son, Explict global Lipschtz solutions to firstorder nonlinear partial differential equations, Viet.J.Math, 27(2) (1999) 93-114

[13] T.D. VAn, N. HoAng, M.Tsuji, On Hopf's formula for Lipschitz solutions of the Cauchy problem for Hamilton-Jacobi equations, Nonlinear Anal. 29 (10) (1997) 11451159.

[14] T.D. Van, M.Tsuji, N.D. Thai Son, The characteristic method and its generalizations for first order nonlinear PDEs, Chapman \& Hall/CRC, London/Boca Raton, $F l, 2000$

[15] Y. ZhaO, T. TANG AND J. WAng , Regularity and global structure of solutions to Hamilton-Jacobi equations I. Convex Hamiltonians, J. Hyperbol. Differ. Eq. 5(3) (2008) 1-18. 